\title{
Introduction to the RE'16 best papers special issue
}

This special issue of the Requirements Engineering Journal contains extended versions of selected papers from the 24th IEEE International Requirements Engineering Conference (RE'16). This continues a long-standing collaboration between RE and REJ to invite authors of the best papers presented at the conference to submit extended papers to reach the wider journal readership. As program chairs of RE'16, it is our pleasure to introduce these papers.

The conference was held in the city of Beijing, China, on September 12-16, 2016, hosted by the General Chair Zhi Jin from Peking University. 216 people from 24 countries attended the conference and its associated tutorials, doctoral symposium and workshops. The conference featured enlightening keynotes by Jan Bosch, Yu Zheng, and Robyn Lutz, presentations of 22 papers in the research track, 11 papers in the industry track, and 11 papers in the RE@Next! track. These papers were selected from 79, 24, and 39 submissions for each track, respectively. Carlos Henrique C. Duarte and Christof Ebert were Program Chairs for the industry track and Travis Breaux and Anna Perini for the RE@Next track. We thank them and all 87 reviewers in the three tracks for the quality of their reviews and feedback to authors.

For this special issue, we invited submission from the authors of the best paper in the industry track and from authors of the 5 research track papers that received the most positive support during the conference reviewing process. We received 4 submissionsAll submitted papers underwent rigorous additional review by three reviewers in accordance with the REJ review process before acceptance to this special issue.

The first paper is "Advancing Viewpoint Merging in Requirements Engineering: A Theoretical Replication and Explanatory Study" by Charu Khatwani, Xiaoyu Jin, Nan Niu, Amy Koshoffer, Linda Newman, and Juha Savolainen. This paper is an extended version of the paper that won the Best Research Paper Award at the conference. The authors present a replication of a study published in RE 2005 that observed that richer domain understanding resulted from viewpoint-based modeling. The replication had two objectives. First, to reproduce the results observed in the base study. Second, to include an evolving factor (namely, the support of a requirements modeling tool) when testing the underlying theory. The replication confirmed the studies of the former study and showed the time saving enabled by the tool.
The second paper is "Extracting Conceptual Models from User Stories with Visual Narrator" by Garm Lucassen, Marcel Robeer, Fabiano Dalpiaz, Jan Martijn E.M. van der Werf, and Sjaak Brinkkemper. Visual Narrator is a tool implementing eleven heuristics to convert a set of user stories into a conceptual model. The proposal is motivated by the need of overcome the limitations of natural language specifications when it comes to detect dependencies between requirements, redundancies and inconsistencies. The approach achieves similar recall and precision as state-of-the-art tools. As a result, Visual Narrator can be an enabler to promote the adoption of conceptual models for discussing about requirements.

The third paper is "Challenges of Working with Artifacts in Requirements Engineering and Software Engineering" by Parisa Ghazi and Martin Glinz. Managing requirements involves manipulating a large number of requirements artifacts such textual requirements, diagrams, sketches, glossary entries and their relations to many other software development artifacts such as design documents, test cases, and change requests. What challenges do software practitioners face when manipulating such large volume of information? Are current tools adequate for the job? In this paper, the authors present the results of an exploratory study where they interviewed 29 practitioners about their current practices. The paper is a must-read for anyone developing requirements engineering and project management tools. Other readers will nod in agreement when recognizing some of the challenges they face themselves (e.g. diagrams too large for the screen, having to work across multiple applications concurrently) and the sometimes convoluted workarounds they have to use to address them.

The fourth paper is "An Exploratory Study of Twitter Messages about Software Applications" by Emitza Guzman, Rana Alkadhi, and Norbert Seyff. This paper is among the first to be published in a promising new line of research that explores the possibilities of learning useful requirements information by mining vast amount of online discussions. Here, the authors focus on analysing twitter messages with the aim of filtering out and classifying messages about particular software applications of interest. For this paper, the authors have analysed over 10 million tweets about 30 different applications. The paper presents important initial results in a rapidly growing research area that might some day radically transform our requirements elicitation practices. 
In addition to these four research papers, we invited the authors of the Best Industry Paper Award in the industry track to submit a "viewpoint" article. The result was the paper "Effectiveness of Focused Mentoring to Improve Requirements Engineering Industrial Practice" by Sarah Gregory and John Terzakis. The authors reflect on their latest experience with their Requirements Authors Mentoring Program (RAMP). In large organizations such as Intel where both authors work, requirements authors are rarely requirements engineering experts and writing requirements is only a small part of their job. With very large numbers of requirements authors spread over different locations over the world, training people in writing high-quality requirements is a challenge. In this paper, the authors describe their experience in setting up and growing a program to recruit and train mentors to help requirements authors improve the quality of their requirements. Requirements mentors help authors by reviewing their requirements and show them how to use structured natural language templates such as EARS and Planguage for writing functional and quality requirements, respectively. Whether you are a researcher or practitioner, you will benefit greatly by reading the authors' practical experience on how to recruit and grow a network of requirements enthusiasts within a large, geographically distributed organization.

We thank the authors and reviewers of the invited papers for their contributions to this special issue. We are also grateful to Peri Loucopoulos, the REJ Editor in Chief, and the REJ editorial team for their assistance in making this special issue possible and for continuing the collaboration between RE and REJ. 